\title{
Transcatheter Arterial Embolization for Shock Caused by Intratumoral Hemorrhaging in Neurofibromatosis Type 1: A Report of Two Cases
}

\author{
Hiroto Saijo Kenji Hayashida Shin Morooka Kaoru Kuwabara \\ Masaki Fujioka \\ Department of Plastic and Reconstructive Surgery, National Hospital Organization, \\ Nagasaki Medical Center, Ohmura City, Japan
}

\section{Key Words}

Neurofibromatosis type 1 - Transcatheter arterial embolization · Intratumoral hemorrhage

\begin{abstract}
Neurofibromatosis type 1 (NF-1) is an autosomal dominant disorder characterized by caféau-lait spots and neurofibroma. Vascular NF-1 lesions are rare, but bleeding from such lesions can sometimes cause lethal complications because surgical hemostasis is difficult to achieve due to the fragile nature of the surrounding blood vessels and soft tissue. In recent years, some reports have suggested that transcatheter arterial embolization (TAE) is an effective treatment for vascular NF-1 lesions. We report the cases of $2 \mathrm{NF}-1$ patients who developed intratumoral hemorrhaging and were successfully treated with TAE.
\end{abstract}

(C) 2014 S. Karger AG, Basel

\section{Introduction}

Neurofibromatosis type 1 (NF-1), which was previously known as von Recklinghausen disease, is an autosomal dominant disorder linked to the proximal portion of the long arm of chromosome 17 that affects 1 in 3,000 individuals [1]. The protein generated by this disorder is called neurofibromin. Neurofibromin exhibits tumor suppressor activity; i.e., it controls the functions of the Ras protein, and is considered to cause various lesions including neurofibromas by inducing abnormal cytostatic activity. However, in recent years it has also been suggested to act via non-Ras-based pathways; thus, its mechanisms of action require 
Saijo et al.: Transcatheter Arterial Embolization for Shock Caused by Intratumoral Hemorrhaging in Neurofibromatosis Type 1: A Report of Two Cases

further clarification. The major clinical features of NF-1 are café-au-lait spots, dermal and plexiform neurofibromas, and skeletal abnormalities. The incidence of vascular conditions associated with NF-1, including stenoses, aneurysms, and arteriovenous malformations, is about 3.6\% [2]. However, NF-1 vasculopathy-related complications are associated with increased morbidity and mortality, particularly among younger patients $[3,4]$.

\section{Case Report}

Case 1

A 77-year-old man fell off his bicycle and bruised the back of his head. He was examined at a local hospital. A computed tomography (CT) scan revealed a hemorrhage in the occipital region so he was admitted to the hospital, where he went into shock and was transferred to our hospital $9 \mathrm{~h}$ after the injury.

On examination, he was found to be alert, his blood pressure was 91/54 mm $\mathrm{Hg}$, his pulse rate was 126 beats/min, and his oxygen saturation level was $100 \%$ whilst breathing through a face mask (5 liter/min). Anemia of the palpebral conjunctiva subsequently developed. Severe swelling and tenderness were observed from the left occipital region to the left side of the neck (fig. 1). Laboratory analysis revealed a blood hemoglobin level of 6.2 $\mathrm{g} / \mathrm{dl}$, but this recovered to $10.0 \mathrm{~g} / \mathrm{dl}$ after the transfusion of 14 units of erythrocytes. We immediately performed a contrast-enhanced CT scan. However, during the scan a mass in the patient's neck suddenly began to swell and obstruct his airway. He underwent emergency intubation. A second contrast-enhanced CT scan revealed an intratumoral hemorrhage, extending from the lower jaw to the left side of the neck and the displacement of the trachea to the right (fig. 2). Thus, he immediately underwent transcatheter arterial embolization (TAE). A left vertebral artery angiogram demonstrated that the branches of the vertebral artery extending into the rear of the neck had expanded and proliferated markedly, and extravasation of contrast material was detected in the peripheral branches (fig. 3). We superselectively embolized the artery using an infusion catheter before injecting gelatin sponge. A subsequent left subclavian artery angiogram depicted extravasation of contrast material in the peripheral branches of the transverse cervical artery. We embolized the artery by injecting an emulsion of n-butyl-2-cyanoacrylate and iodized oil at a ratio of 1:4 (Histoacryl/Lipiodol) (fig. 3).

The TAE was successful, and the hemorrhage gradually reduced in size. Since the narrowing of the patient's airway improved, the patient was extubated at 8 days after admission. Subsequently, the hemorrhagic region exhibited autolysis, and the ulcers on the back of the patient's head and left shoulder were drained. He underwent a free skin grafting at 21 days after admission. The patient's wounds completely healed, and he was discharged at 41 days after admission (fig. 4).

Case 2

A 33-year-old woman developed a huge pseudoneuroma in her right thigh; she had undergone six tumor resection operations and an above-the-knee amputation. Suddenly, she complained of pain in her right thigh. Her blood pressure was $89 / 77 \mathrm{~mm} \mathrm{Hg}$, and her pulse rate was 128 beats/min. Severe pain and swelling developed in her right thigh (fig. 5). Her blood pressure was immediately increased by fluid administration. A contrast-enhanced CT imaging showed an aneurysm, which had ruptured, resulting in a huge intratumoral hemorrhage (fig. 6). We immediately performed TAE, and extravasation of contrast material was detected in the peripheral branches of the lateral circumflex femoral artery (fig. 7). We 
embolized the contrast material leakage sites by injecting an emulsion of n-butyl-2cyanoacrylate and iodized oil at a ratio of 1:4 (Histoacryl/Lipiodol). After the TAE had been completed, the patient's blood hemoglobin level fell to $6.2 \mathrm{~g} / \mathrm{dl}$, but it was successfully restored via the transfusion of two units of erythrocytes. The patient was discharged without complications.

\section{Discussion}

Greene et al. [5] classified the arterial lesions associated with neurofibromatosis into two types. In the first type, larger vessels such as the aorta, carotid artery, etc., are surrounded by perivascular neurofibromatous or ganglioneuromatous tissue, leading to intimal proliferation, thinning of the tunica media, and the fragmentation of elastic tissue, resulting in aneurysm formation or stenosis. In the second type, smaller vessels are affected by dysplasia rather than neural malformations.

Pereira et al. [6] hypothesized that the vascular lesions that develop in NF-1 are caused by neurofibromin stimulating the proliferation of smooth muscle cells and endothelial cells and impairing their destruction, leading to increases in the vulnerability of the vessel wall and the extent of stenosis and a reduction in the integrity of the endothelial layer cell. In addition to the blood vessel fragility mentioned above, as significant angiogenesis occurs within diffuse neurofibromas it is often difficult to achieve hemostasis using sutures. Miura et al. [7] reported 12 cases of spontaneous hemothorax involving Japanese NF-1 patients. In their report, surgery was performed in 10 patients, and half of the patients died due to blood loss despite treatment. In addition, pressure hemostasis is even difficult to achieve in cases involving bleeding from soft tissue close to the body surface due to the fragile nature of the skin and connective tissue in NF-1 patients.

In recent years, there has been a tendency to use TAE to treat intrathoracic hemorrhaging in NF-1 [8-10]. In such cases, TAE is performed as follows: a catheter is inserted under fluoroscopic guidance, the leakage site is confirmed using contrast-enhanced angiography, and embolization is performed using an embolic material and a coil. TAE is considered to be minimally invasive and has the advantage of only embolizing selected arteries. The present two cases involved patients that went into shock due to intratumoral hemorrhaging, but it was possible to perform TAE safely by first stabilizing the patients' with blood transfusions and intubating them for airway management. In case 1, partial necrosis of the tumor occurred after TAE, but the wound was successfully closed with a free skin graft. Bleeding was not a problem as the main blood vessels leading to the tumor had been embolized. Some previous studies have reported that TAE was effective against hemorrhaging in the neck, chest cavity, or abdomen in NF-1 patients. Based on the above points, we recommend performing TAE in cases of intratumoral hemorrhaging involving the extremities or soft tissue close to the body surface because surgical hemostasis is very difficult to achieve.

\section{Conclusion}

We have successfully used TAE to treat 2 NF-1 patients that fell into hemorrhagic shock. Arterial lesions are rare in NF-1 patients, but can be life-threatening if major bleeding occurs. TAE is minimally invasive, and thus, should be considered to be the first-choice treatment in such cases. 
Saijo et al.: Transcatheter Arterial Embolization for Shock Caused by Intratumoral Hemorrhaging in Neurofibromatosis Type 1: A Report of Two Cases

\section{References}

1 Oderich GS, Sullivan TM, Bower TC, Gloviczki P, Miller DV, Babovic-Vuksanovic D, et al: Vascular abnormalities in patients with neurofibromatosis syndrome type I: clinical spectrum, management, and results. J Vasc Surg 2007;46:475-484.

-2 Brasfield RD, Das Gupta TK: Von Recklinghausen's disease: a clinicopathological study. Ann Surg 1972;175:86-104.

-3 Rasmussen SA, Yang Q, Friedman JM: Mortality in neurofibromatosis 1: an analysis using U.S. death certificates. Am J Hum Genet 2001;68:1110-1118.

-4 Lasater EA, Li F, Bessler WK, Estes ML, Vemula S, Hingtgen CM, et al: Genetic and cellular evidence of vascular inflammation in neurofibromin-deficient mice and humans. J Clin Invest 2010;120:859-870.

-5 Greene JF, Fitzwater JE, Burgess J: Arterial lesions associated with neurofibromatosis. Am J Clin Pathol 1974;62:481-487.

6 Pereira VM, Geiprasert S, Krings T, Caldas JG, Toulgoat F, Ozanne A, et al: Extracranial vertebral artery involvement in neurofibromatosis type I. Report of four cases and literature review. Interv Neuroradiol 2007;13:315-328.

7 Miura H, Taira O, Uchida O, Usuda J, Hirai S, Kato H: Spontaneous haemothorax associated with von Recklinghausen's disease: review of occurrence in Japan. Thorax 1997;52:577-578.

-8 Hieda M, Toyota N, Kakizawa H, Hirai N, Tachikake T, Yahiro Y, et al: Endovascular therapy for massive haemothorax caused by ruptured extracranial vertebral artery aneurysm with neurofibromatosis Type $1 . \mathrm{Br}$ J Radiol 2007;80:e81- e84.

-9 Uzuka T, Ito T, Koyanagi T, Maeda T, Tabuchi M, Kawaharada N, et al: Giant intercostal aneurysm complicated by Stanford type B acute aortic dissection in patients with type 1 neurofibromatosis. J Cardiothorac Surg 2012;7:38.

10 Hongsakul K, Rookkapan S, Tanutit P, Pakdeejit S, Songjamrat A, Sungsiri J: Spontaneous massive hemothorax in a patient with neurofibromatosis type 1 with successful transarterial embolization. Korean J Radiol 2013;14:86-90.

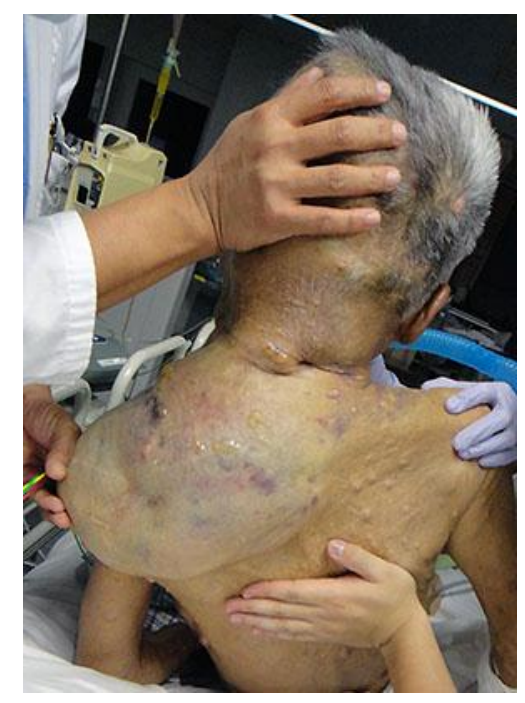

Fig. 1. Case 1: image showing severe swelling and pain in the patient's neck. 
Saijo et al.: Transcatheter Arterial Embolization for Shock Caused by Intratumoral Hemorrhaging in Neurofibromatosis Type 1: A Report of Two Cases

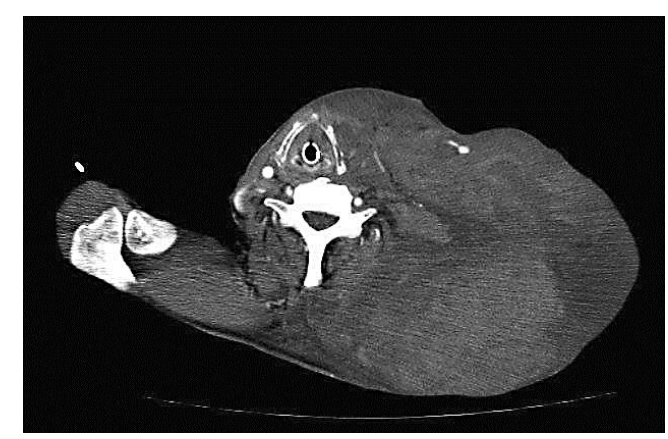

Fig. 2. CT scan obtained after intubation showing an intratumoral hemorrhage and the displacement of the trachea to the right.
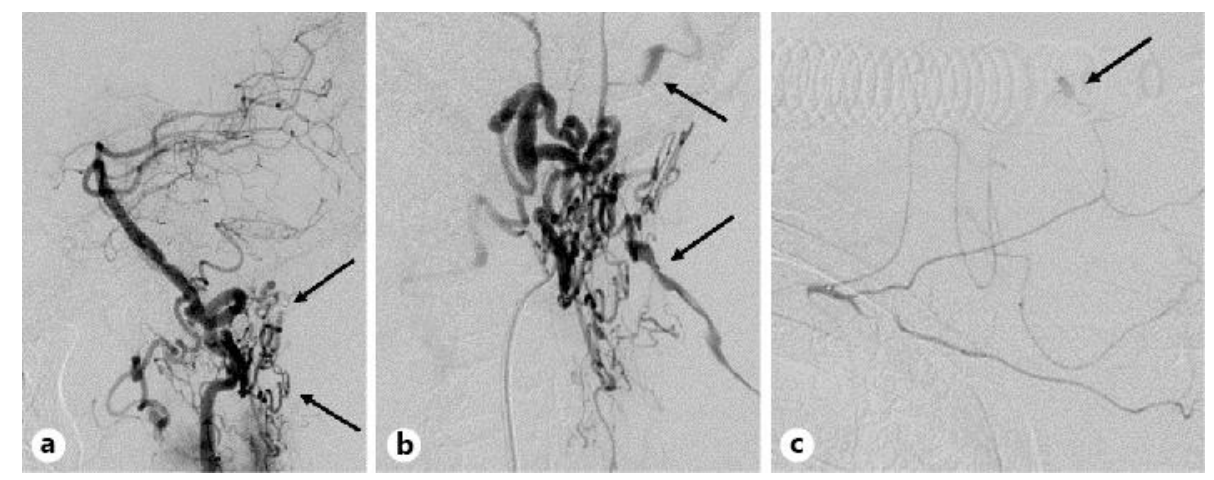

Fig. 3. a Left vertebral artery angiogram (lateral view) showing angiogenesis in the tumor (arrows). b Left vertebral artery angiogram (frontal view) showing extravasation of contrast material in the peripheral branches of the vertebral artery (arrows). c Left subclavian artery angiogram showing extravasation of contrast material in the transverse cervical artery (arrow). 


\section{Case Reports in Dermatology}

\section{Case Rep Dermatol 2014;6:59-65 \\ DOI: $10.1159 / 000360216$}

Saijo et al.: Transcatheter Arterial Embolization for Shock Caused by Intratumoral
Hemorrhaging in Neurofibromatosis Type 1: A Report of Two Cases
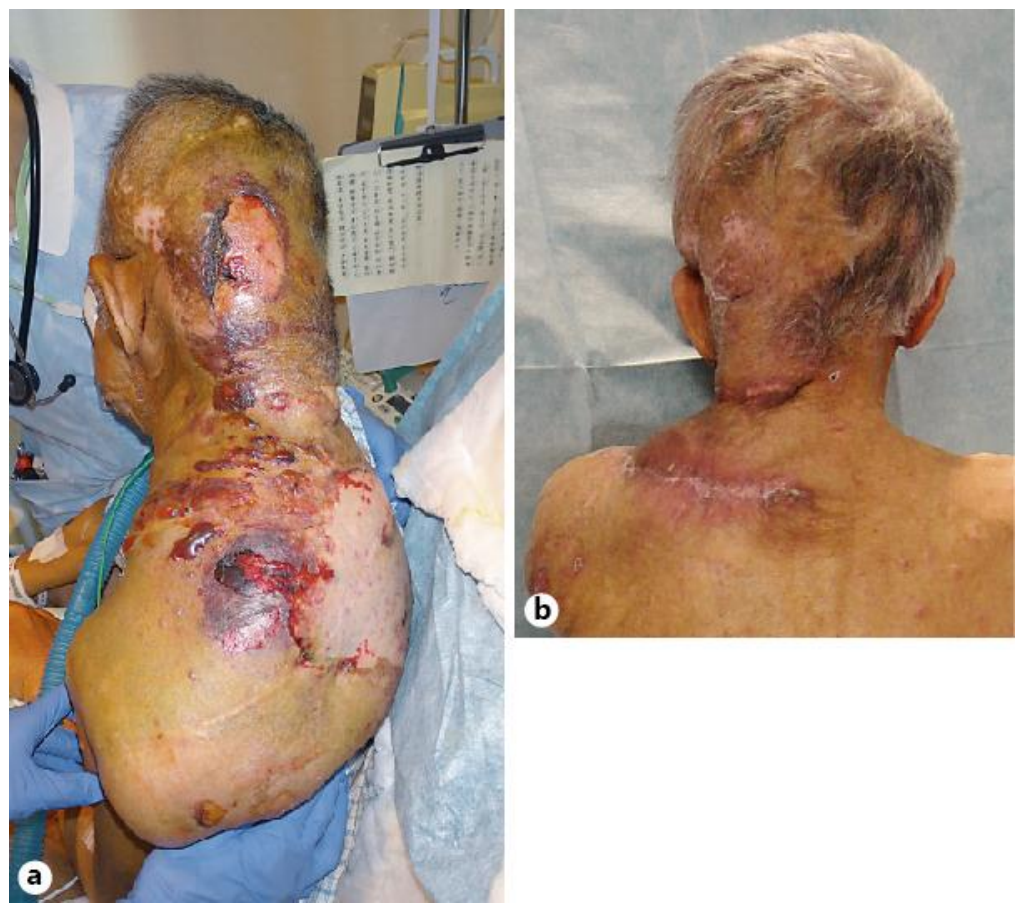

Fig. 4. a Image showing an ulcer on the hematoma on 21 days after admission. $\mathbf{b}$ The patient's wounds have completely healed 41 days after admission.

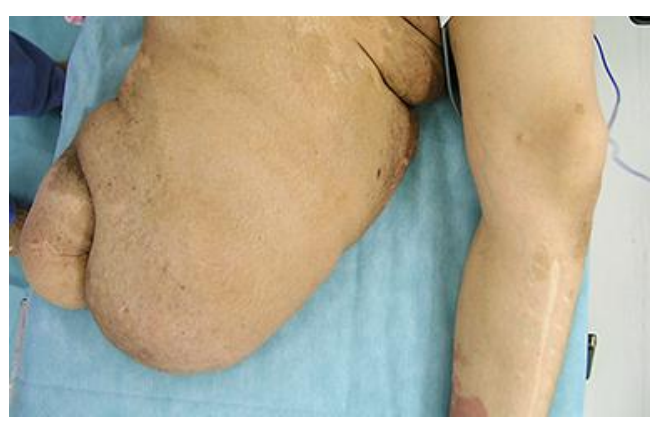

Fig. 5. Case 2: image of the patient showing severe swelling and pain in her right thigh. 


\section{Case Reports in Dermatology}

Case Rep Dermatol 2014;6:59-65

DOI: $10.1159 / 000360216$

Saijo et al.: Transcatheter Arterial Embolization for Shock Caused by Intratumoral

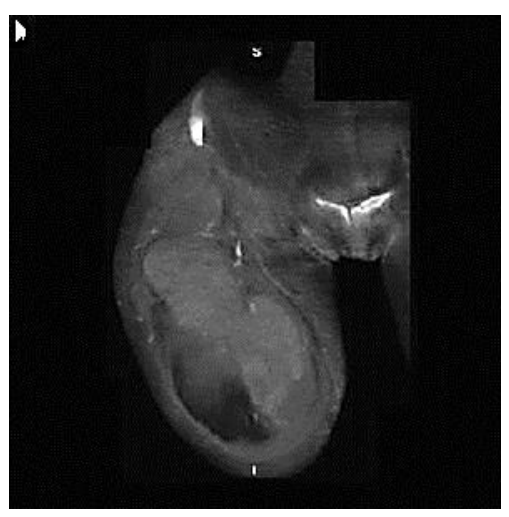

Fig. 6. CT scan showing a huge intratumoral hemorrhage in the patient's right thigh.

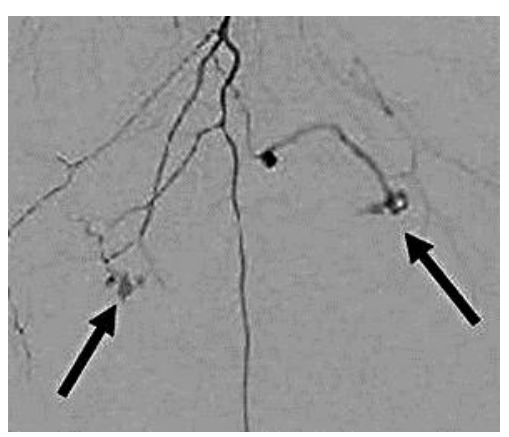

Fig. 7. Right lateral circumflex femoral artery angiogram showing an aneurysm and extravasation of contrast material. 